PROCEEDINGS OF THE

AMERICAN MATHEMATICAL SOCIETY

Volume 137, Number 7, July 2009, Pages 2181-2189

S 0002-9939(09)09760-3

Article electronically published on January 26, 2009

\title{
CONSTRUCTING BIG INDECOMPOSABLE MODULES
}

\author{
ANDREW CRABBE AND JANET STRIULI
}

(Communicated by Bernd Ulrich)

\begin{abstract}
Let $R$ be local Noetherian ring of depth at least two. We prove that there are indecomposable $R$-modules which are free on the punctured spectrum of constant, arbitrarily large, rank.
\end{abstract}

\section{INTRODUCTION}

A fruitful approach to the study of a commutative ring is to understand the category of its finitely generated modules. Over zero dimensional rings, it is feasible to understand the entire category by classifying its indecomposable objects; however, over larger rings, this classification is not a viable possibility. A more tractable category that still encodes important information about the ring is that of maximal Cohen-Macaulay modules. For example, finiteness conditions on the category of maximal Cohen-Macaulay modules detect certain types of singularities [1], 3]. Such finiteness conditions cannot necessarily be applied to other categories of modules, as one can build arbitrarily big indecomposable modules which are not maximal Cohen-Macaulay.

Our interest was raised by work of W. Hassler, R. Karr, L. Klingler and R. Wiegand. Indeed, they build indecomposable modules of arbitrarily large rank over hypersurface singularities with positive dimension which are not $A_{1}$-singularities ([10]), and over rings which are not homomorphic images of Dedekind-like rings ([7], [8], 9]). While over hypersurfaces, the constructed indecomposable modules are free of constant rank on the punctured spectrum, in the case of rings which are not homomorphic images of Dedekind-like rings, the indecomposable modules are only known to be locally free at a finite set of primes. Our main result is the following theorem, which for rings of depth at least two gives indecomposable modules free of arbitrarily large constant rank on the punctured spectrum.

(1.1) Theorem. Let $R$ be a local Noetherian ring of depth at least two and not regular. Given any integer $r$, there exists a short exact sequence of finitely generated R-modules:

$$
0 \rightarrow T \rightarrow X \rightarrow M \rightarrow 0
$$

Received by the editors May 8, 2008, and, in revised form, August 29, 2008

2000 Mathematics Subject Classification. Primary 13H10, 13C14, 13 E05.

Key words and phrases. Indecomposable modules, maximal Cohen-Macaulay modules, rank. The second author was partially supported by NSF grant DMS 0201904.

(C)2009 American Mathematical Society Reverts to public domain 28 years from publication 
in which $T$ has finite length, $M$ has positive depth, $X$ is indecomposable, and $M$ and $X$ are free of constant rank on the punctured spectrum, and such a rank is at least $r$. If further $R$ is Cohen-Macaulay, $M$ can be chosen to be maximal Cohen-Macaulay.

For every $R$-module $N$, denote by $N^{(r)}$ the direct sum of $r$ copies of $N$. When the ring is complete of dimension at least two (with no restriction on the depth), we even prove that for every integer $r$ and indecomposable module $M$, which is free of constant rank on the punctured spectrum and has positive depth, there exists a short exact sequence of $R$-modules $0 \rightarrow T \rightarrow X \rightarrow M^{(r)} \rightarrow 0$, where $X$ is indecomposable and $T$ has finite length. One cannot relax the condition on the depth as it is known that the rank of an indecomposable module is at most two for certain rings of depth one, for example $A_{1}$-singularity ([15, [14, [13]).

Theorem 1.1 is particularly interesting when the ring is Cohen-Macaulay of bounded Cohen-Macaulay type, by which we mean that there is an upper bound on the minimal number of generators for any indecomposable maximal CohenMacaulay module. Theorem 1.1 says that one cannot possibly hope for such a bound on indecomposable modules that are not maximal Cohen-Macaulay.

\section{Preliminaries}

In the following, $(R, \mathfrak{m}, \mathrm{k})$ is a local Noetherian ring and all modules are finitely generated. Short exact sequences are identified with elements of the module $\operatorname{Ext}_{R}^{1}(-,-)$; for details see [17.

(2.1) Actions. If $\alpha$ is an element $\operatorname{Ext}_{R}^{1}(M, N)$, and if $f$ is an $R$-homomorphism in $\operatorname{Hom}_{R}\left(M^{\prime}, M\right)$, then $\alpha f$ denotes the element in $\operatorname{Ext}_{R}^{1}\left(M^{\prime}, N\right)$ obtained from the pullback of $\alpha$ via $f$, as shown in the following diagram:

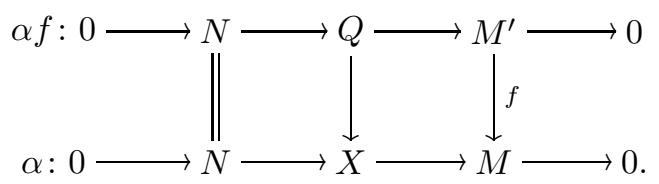

Set $\mathcal{B}=\operatorname{Hom}_{R}(M, M)$; if $M=M^{\prime}$, then the above action gives $\operatorname{Ext}_{R}^{1}(M, N)$, a structure of a $\mathcal{B}$-module. To avoid any confusion we write $\operatorname{Ext}_{R}^{1}(M, N)_{\mathcal{B}}$.

In order to present our proof, we need to recall a result from [10] which gives the tool for constructing indecomposable modules.

(2.2) Indecomposable modules [10, Theorem 2.3]. Let $M$ be a finitely generated $R$-module of positive depth and let $T$ be an indecomposable finitely generated $R$ module of finite length. Set $\mathcal{B}=\operatorname{Hom}_{R}(M, M)$. Suppose there exists an element $\alpha \in \operatorname{Ext}_{R}^{1}(M, T)$ such that $\operatorname{ann}_{\mathcal{B}}(\alpha) \subseteq J(\mathcal{B})$, where $J(-)$ denotes the Jacobson radical. If $0 \rightarrow T \rightarrow X \rightarrow M \rightarrow 0$ represents $\alpha$, then $X$ is indecomposable.

When the ring is complete, the following lemma gives us the element $\alpha$.

(2.3) Lemma. Let $R$ be a complete ring and let $M, N$ be finitely generated $R$ modules. Suppose that $M$ is indecomposable and set $\mathcal{A}=\operatorname{Hom}_{R}(M, M)$. Let $g_{1}, \ldots, g_{r}$ be part of the minimal generating set for $\operatorname{Ext}_{R}^{1}(M, N)$ as a right $\mathcal{A}$ module. Set $\mathcal{C}=\mathrm{M}_{r \times r}(\mathcal{A})$, the ring of $r \times r$ matrices with entries in $\mathcal{A}$. Define $\rho: \mathcal{C}_{\mathcal{C}} \rightarrow\left(\operatorname{Ext}_{R}^{1}(M, N)^{(r)}\right)_{\mathcal{C}}$ to be the right $\mathcal{C}$-module homomorphism such that for every $\gamma \in \mathcal{C}, \rho(\gamma)=\left(g_{1}, \ldots, g_{r}\right) \gamma$, via matrix multiplication. Then $\operatorname{ker}(\rho) \subseteq J(\mathcal{C})$. 
In particular there exists an element $\alpha \in \operatorname{Ext}_{R}^{1}\left(M^{(r)}, N\right)$ such that $\operatorname{ann}_{\mathcal{B}}(\alpha) \subseteq$ $J(\mathcal{B})$, where $\mathcal{B}:=\operatorname{Hom}_{R}\left(M^{(r)}, M^{(r)}\right)$.

Proof. Let $\phi \in \operatorname{ker}(\rho)$ and write $\phi=\left(a_{i j}\right)$, where $a_{i j} \in \mathcal{A}$. For each $j=1, \ldots, r$, the equality $\sum_{i=1}^{r} g_{i} a_{i j}=0$ holds. Since $M$ is indecomposable and $R$ is complete, $\mathcal{A}$ is a local ring with the Jacobson radical $J(\mathcal{A})$ as its unique maximal two-sided ideal. By the assumption on $g_{1}, \ldots, g_{r}$, it follows that $a_{i j} \in J(\mathcal{A})$ for each $i$ and each $j$ [18, Lemma 4.43]. Since $J(\mathcal{C})=\mathrm{M}_{r \times r}(J(\mathcal{A}))$, the element $\phi$ belongs to $J(\mathcal{C})$; see, for example, [11, Exercise 13, page 433].

For the "in particular" statement, there are isomorphisms $\phi: \operatorname{Ext}_{R}^{1}\left(M^{(r)}, N\right) \rightarrow$ $\operatorname{Ext}_{R}^{1}(M, N)^{(r)}$ (see 4.1) and $\psi: \mathcal{B} \rightarrow \mathcal{C}$ (see 4.2) which are compatible with the action of $\mathcal{C}$ on $\operatorname{Ext}_{R}^{1}(M, N)^{(r)}$ (matrix multiplication) and the action of $\mathcal{B}$ on $\operatorname{Ext}_{R}^{1}\left(M^{(r)}, N\right)$ (see 2.1); for details see Proposition 4.3. The element $\alpha=$ $\phi^{-1}\left(\left(g_{1}, \ldots, g_{r}\right)\right)$ satisfies $\operatorname{ann}_{\mathcal{B}}(\alpha)=\operatorname{ker} \rho \subseteq J(\mathcal{B})$.

The following theorem is the first step in proving Theorem 1.1. In the rest of the paper, given an $R$-module $M$, we denote by $\nu_{R}(M)$ the minimal number of generators of $M$ as an $R$-module.

(2.4) Theorem. Let $(R, \mathfrak{m}, \mathrm{k})$ be a local complete Noetherian ring and let $M$ be an indecomposable $R$-module of positive depth. Assume that

$$
\limsup _{n \rightarrow \infty} \nu_{R}\left(\operatorname{Ext}_{R}^{1}\left(M, R / \mathfrak{m}^{n}\right)\right)=\infty .
$$

Let $r$ be an integer. Then there exists a short exact sequence

$$
\alpha: 0 \rightarrow R / \mathfrak{m}^{n} \rightarrow X \rightarrow M^{(r)} \rightarrow 0,
$$

such that $X$ is an indecomposable $R$-module.

Proof. Let $\mathcal{A}$ be the finitely generated $R$-algebra $\operatorname{Hom}_{R}(M, M)$. Suppose that $\nu_{R}(\mathcal{A})=h$. Then the following holds:

$$
\begin{gathered}
\nu_{\mathcal{A}}\left(\operatorname{Ext}_{R}^{1}\left(M, R / \mathfrak{m}^{n}\right)\right) \geq \nu_{R}\left(\operatorname{Ext}_{R}^{1}\left(M, R / \mathfrak{m}^{n}\right)\right) / h, \text { and so } \\
\limsup _{n \rightarrow \infty} \nu_{\mathcal{A}}\left(\operatorname{Ext}_{R}^{1}\left(M, R / \mathfrak{m}^{n}\right)\right)=\infty .
\end{gathered}
$$

For a given integer $r$, choose $n$ such that $\nu_{\mathcal{A}}\left(\operatorname{Ext}_{R}^{1}\left(M, R / \mathfrak{m}^{n}\right)\right)>r$. Let $g_{1}, \ldots, g_{r}$ be part of a minimal generating set of $\operatorname{Ext}_{R}^{1}\left(M, R / \mathfrak{m}^{n}\right)$ as an $\mathcal{A}$-module. By Lemma 2.3. there exists $\alpha \in \operatorname{Ext}_{R}^{1}\left(M^{(r)}, R / \mathfrak{m}^{n}\right)$ such that $\operatorname{ann}_{\mathcal{B}}(\alpha) \subseteq J(\mathcal{B})$, where $\mathcal{B}:=$ $\operatorname{Hom}_{R}\left(M^{(r)}, M^{(r)}\right)$. Let $\alpha$ be represented by the short exact sequence $0 \rightarrow R / \mathfrak{m}^{n} \rightarrow$ $X \rightarrow M^{(r)} \rightarrow 0$. By (2.2), the $R$-module $X$ is indecomposable.

The next step is to find modules for which equation (11) in Theorem 2.4 is satisfied.

(2.5) Generalized Hilbert polynomials. Let $M$ be a finitely generated $R$ module. It is known that the lengths of the $R$-modules $\operatorname{Ext}_{R}^{1}\left(M, R / \mathfrak{m}^{n} R\right)$ are given by a polynomial $\xi_{M, \mathfrak{m}}(n)$ for all $n$ large enough (see [12], [16]). In [4, the authors give an exact formula for the degree of this polynomial; in particular when $M$ has constant rank on the punctured spectrum, the degree is $\operatorname{dim} R-1$.

Often if a module $M$ is free on the punctured spectrum, then it has constant rank. This is the result of the following lemma, which relies on Hartshorne's Connectedness Theorem: if the depth of a local Noetherian ring is at least two, then the punctured spectrum is connected. 
(2.6) Lemma. Let $R$ be a local Noetherian ring of depth at least two. Let $M$ be an $R$-module free in the punctured spectrum. Then $M$ is free of constant rank in the punctured spectrum.

Proof. For $i=1, \ldots, n$, let $\mathfrak{p}_{i}$ denote the minimal primes of $R$, and set $t=$ $\max \left\{\operatorname{rank}_{R_{\mathfrak{p}_{i}}}\left(M_{\mathfrak{p}_{i}}\right) \mid i=1, \ldots, n\right\}$. We wish to show that $M$ is free of constant rank on the punctured spectrum and we will proceed by contradiction. Without loss of generality, we may assume that $\operatorname{rank}_{R_{\mathfrak{p}_{i}}}\left(M_{\mathfrak{p}_{i}}\right)=t$ for all $i \leq s$ and $\operatorname{rank}_{R_{\mathfrak{p}_{i}}}\left(M_{\mathfrak{p}_{i}}\right)<t$ for $s<i \leq n$. Set $I=\bigcap_{i=1}^{s} \mathfrak{p}_{i}$ and $J=\bigcap_{i=s+1}^{n} \mathfrak{p}_{i}$. For every ideal $L$, let $\mathcal{V}(L)$ be the closed set in $\operatorname{Spec} R$ which gives the support of $R / L$. We claim that the two open sets Spec $R \backslash \mathcal{V}(I)$ and Spec $R \backslash \mathcal{V}(J)$ disconnect the punctured spectrum Spec $R \backslash\{\mathfrak{m}\}$, contradicting Hartshorne's Connectedness Theorem; see $[5$. To prove the claim, it is enough to show that $\{\mathfrak{m}\}=\mathcal{V}(I) \cap \mathcal{V}(J)$, since Spec $R=\mathcal{V}(I) \cup \mathcal{V}(J)$ by the definition of $I$ and $J$. Assume by contradiction that there exists a prime $\mathfrak{q} \neq \mathfrak{m}$ in the set $\mathcal{V}(I) \cap \mathcal{V}(J)$. Then there exists an $i \leq s$ and $j>s$ such that $\mathfrak{p}_{i} \subset \mathfrak{q}$ and $\mathfrak{p}_{j} \subset \mathfrak{q}$. But then $t=\operatorname{rank}_{R_{\mathfrak{p}_{i}}}\left(M_{\mathfrak{p}_{i}}\right)=\operatorname{rank}_{R_{\mathfrak{q}}}\left(M_{\mathfrak{q}}\right)=\operatorname{rank}_{R_{\mathfrak{p}_{j}}}\left(M_{\mathfrak{p}_{j}}\right)<t$.

We end the section with a technical lemma.

(2.7) Lemma. Let $\left\{M_{n}\right\}$ be a family of finitely generated $R$-modules of finite length. Assume that for fixed integers $s>0, a>0$, and $b$, the following hold: $\mathfrak{m}^{s} M_{n}=0$ and $\lambda\left(M_{n}\right) \geq a n+b$, for all $n \gg 0$. Then for any given integer $h$, there exists an $n$ such that $\nu_{R}\left(M_{n}\right)>h$.

Proof. Set $t=\max _{i=0, \ldots, s}\left\{\nu_{R}\left(\mathfrak{m}^{i}\right)\right\}$, let $h$ be a given integer and choose $k>t h$. Choose $n$ large enough so that $\left(a_{1} n+a_{0}\right) / s \geq k$. There is an equality on lengths

$\lambda\left(M_{n}\right)=\lambda\left(M_{n} / \mathfrak{m} M_{n}\right)+\lambda\left(\mathfrak{m} M_{n} / \mathfrak{m}^{2} M_{n}\right)+\cdots+\lambda\left(\mathfrak{m}^{s-2} M_{n} / \mathfrak{m}^{s-1} M_{n}\right)+\lambda\left(\mathfrak{m}^{s-1} M_{n}\right)$, and since $\lambda\left(M_{n}\right) \geq a_{1} n+a_{0}$, there must be an $a<s$ for which $\lambda\left(\mathfrak{m}^{a} M_{n} / \mathfrak{m}^{a+1} M_{n}\right) \geq$ $\left(a_{1} n+a_{0}\right) / s$. This implies

$$
\begin{aligned}
t \nu_{R}\left(M_{n}\right) & \geq \nu\left(\mathfrak{m}^{a}\right) \nu\left(M_{n}\right) \geq \nu\left(\mathfrak{m}^{a} M_{n}\right) \\
& =\lambda\left(\mathfrak{m}^{a} M_{n} / \mathfrak{m}^{a+1} M_{n}\right) \\
& \geq\left(a_{1} n+a_{0}\right) / s \geq k>t h,
\end{aligned}
$$

and therefore $\nu_{R}\left(M_{n}\right)>h$.

\section{MAin Results}

In this section we prove Theorem 1.1 from the introduction. We first handle the case where the ring is complete. If $M$ is an $R$-module, we denote by $\Omega_{R}^{i}(M)$ the $i$ th syzygy of $M$ in a minimal free resolution.

(3.1) Theorem. Let $(R, \mathfrak{m}, \mathrm{k})$ be a local complete ring of dimension at least two. Let $M$ be an indecomposable $R$-module of positive depth that is free of constant rank on the punctured spectrum. Given any integer $r$, there exists an integer $n$ and a short exact sequence

$$
0 \rightarrow R / \mathfrak{m}^{n} \rightarrow X \rightarrow M^{(r)} \rightarrow 0
$$

where $X$ is indecomposable, free on the punctured spectrum with $\operatorname{rank}_{R_{\mathfrak{p}}}\left(X_{\mathfrak{p}}\right) \geq r$, for every prime ideal $\mathfrak{p} \neq \mathfrak{m}$. 
Proof. Since $M$ is free on the punctured spectrum, $\operatorname{Ext}_{R}^{1}\left(M, \Omega_{R}^{1}(M)\right)$ is a module of finite length, so there exists an integer $s$ such that $\mathfrak{m}^{s} \operatorname{Ext}_{R}^{1}\left(M, \Omega_{R}^{1}(M)\right)=0$. Let $\beta$ be the element $0 \rightarrow \Omega_{R}^{1}(M) \rightarrow F \rightarrow M \rightarrow 0$ in $\operatorname{Ext}_{R}^{1}\left(M, \Omega_{R}^{1}(M)\right)$, and let $N$ be an $R$-module. For every element $\alpha \in \operatorname{Ext}_{R}^{1}(M, N)$ there exists a homomorphism $f \in$ $\operatorname{Hom}_{R}\left(\Omega_{R}^{1}(M), N\right)$ such that $\alpha=\beta f$. Therefore $\mathfrak{m}^{s} \subseteq \operatorname{ann}_{R}\left(\operatorname{Ext}_{R}^{1}(M, N)\right)$ for every finitely generated module $N$, and in particular the equality $\mathfrak{m}^{s} \operatorname{Ext}_{R}^{1}\left(M, R / \mathfrak{m}^{n}\right)=0$ holds for every integer $n>0$. By hypothesis on the dimension of the ring and by (2.5), the lengths of the modules $\operatorname{Ext}_{R}^{1}\left(M, R / \mathfrak{m}^{n}\right)$ are given by a polynomial of degree at least one. By Lemma 2.7, $\lim \sup _{n \rightarrow \infty} \nu_{R}\left(\operatorname{Ext}_{R}^{1}\left(M, R / \mathfrak{m}^{n}\right)\right)=\infty$, and by Theorem 2.4, for every positive integer $r$ there exists a short exact sequence

$$
0 \rightarrow R / \mathfrak{m}^{n} \rightarrow X \rightarrow M^{(r)} \rightarrow 0
$$

where $X$ is indecomposable. For every prime ideal $\mathfrak{p} \in \operatorname{Spec}(R)$, the module $X_{\mathfrak{p}}$ is isomorphic to $M_{\mathfrak{p}}^{(r)}$, which is free by hypothesis.

We now remove the assumption on $R$ being complete and prove Theorem 1.1 from the introduction. Given a finitely generated $R$-module $P$, denote by $H_{\mathfrak{m}}^{0}(P)$ the largest submodule of finite length of $P$.

Proof. Let $d=\operatorname{dim} R$ and let $N$ be an indecomposable summand of the $R$-module $\Omega_{R}^{d}(\mathrm{k}) / H_{\mathfrak{m}}^{0}\left(\Omega_{R}^{d}(\mathrm{k})\right)$. Write $N \otimes \widehat{R}=\bigoplus_{i=1}^{k} N_{i}$, where the $N_{i}$ are indecomposable summands of $\Omega_{\widehat{R}}^{d}(\mathrm{k}) / H_{\mathfrak{m}}^{0}\left(\Omega_{\widehat{R}}^{d}(\mathrm{k})\right)$. The modules $N_{i}$ have positive depth and are free on the punctured spectrum; thus by Lemma 2.6 and by the hypothesis on the depth of the ring, they have constant rank. Fix an integer $n$. For each $i=1, \ldots, k$, by Theorem 3.1, there exist an integer $n_{i}$ and a short exact sequence

$$
\alpha_{i}: 0 \rightarrow R / \mathfrak{m}^{n_{i}} \rightarrow Y_{i} \rightarrow N_{i}^{(n)} \rightarrow 0
$$

where $Y_{i}$ is an indecomposable $\widehat{R}$-module. Consider the sequence

$$
\bigoplus_{i=1}^{k} \alpha_{i}: \quad 0 \rightarrow \bigoplus_{i=1}^{k} R / \mathfrak{m}^{n_{i}} \rightarrow \bigoplus_{i=1}^{k} Y_{i} \rightarrow \bigoplus_{i=1}^{k} N_{i}^{(n)} \rightarrow 0
$$

The right-hand and left-hand modules are extended 1 Since the length of $\operatorname{Ext}_{R}^{1}\left(N^{(n)}, \bigoplus_{i=1}^{k} R / \mathfrak{m}^{n_{i}}\right)$ is finite, by [6, Proposition 3.2(1)] the middle module is also an extended module, so say $\bigoplus_{i=1}^{k} Y_{i} \cong Y \otimes \widehat{R}$, where $Y$ is a finitely generated $R$-module. In particular, there exists a short exact sequence

$$
0 \longrightarrow \bigoplus_{i=1}^{k} R / \mathfrak{m}^{n_{i}} \longrightarrow Y \longrightarrow N^{(n)} \longrightarrow 0,
$$

where $Y$ is an $R$-module free on the punctured spectrum of constant rank equal to $n \cdot \operatorname{rank}(N)$. If $Y$ is indecomposable, then we are done. If not, since $Y$ has at most $k$ indecomposable summands, we may choose an indecomposable summand $X$ of $Y$ that is free on the punctured spectrum with rank at least $n \cdot \frac{\operatorname{rank}(N)}{k}$. Since $X \otimes \widehat{R}$ is a direct summand of $Y \otimes \widehat{R}$, we can, by possibly reordering the indices, write $X \otimes \widehat{R} \cong \bigoplus_{i=1}^{\ell} Y_{i}$, where $\ell \leq k$. Consider the sequence

$$
\bigoplus_{i=1}^{\ell} \alpha_{i}: \quad 0 \rightarrow \bigoplus_{i=1}^{\ell} R / \mathfrak{m}^{n_{i}} \rightarrow \bigoplus_{i=1}^{\ell} Y_{i} \rightarrow \bigoplus_{i=1}^{\ell} N_{i}^{(n)} \rightarrow 0 .
$$

\footnotetext{
${ }^{1}$ We say that an $\widehat{R}$-module $M$ is extended if there exists an $R$-module $N$ such that $N \otimes \widehat{R} \cong M$.
} 
Since the left-hand and middle modules are extended and since $\operatorname{Hom}_{R}\left(\bigoplus_{i}^{\ell} R / \mathfrak{m}^{n_{i}}, \bigoplus_{i}^{\ell} Y_{i}\right)$ has finite length, by [6, Proposition 3.2(3)] the right-hand module is extended; so say $\bigoplus_{i=1}^{\ell} N_{i}^{(n)} \cong L \otimes \widehat{R}$, where $L$ is a finitely generated $R$ module. Since $L \otimes \widehat{R}$ is a direct summand of $N^{(n)} \otimes \widehat{R}$, by an argument in the proof of [6. Proposition 3.1] $L$ is a direct summand of $N^{(n)}$; hence $\operatorname{depth}_{R} L \geq \operatorname{depth}_{R} N$. Since $\operatorname{depth}_{R} N>0$, the sequence

$$
0 \longrightarrow \bigoplus_{i=1}^{\ell} R / \mathfrak{m}^{n_{i}} \longrightarrow X \longrightarrow 0
$$

satisfies the conclusion of the theorem.

If the ring $R$ is Cohen-Macaulay, then $N$ is a direct summand of $\Omega_{R}^{d}(\mathrm{k})$, which is a maximal Cohen-Macaulay module.

\section{Appendix}

In the following we record some known facts about the $R$-module $\operatorname{Ext}_{R}^{1}(M, N)$, where $M$ and $N$ are two finitely generated $R$-modules. Our main source is [17.

Let $N$ and $M_{i}$, for $i=1, \ldots n$, be finitely generated $R$-modules. In the following, $\mathrm{m}$ denotes an element in $\bigoplus_{i=1}^{n} M_{i}$, and $\mathrm{m}_{j} \in M_{j}$ is the $j$ th component of $\mathrm{m}$. Let $\iota_{j}: M_{j} \rightarrow \bigoplus_{i=1}^{n} M_{i}$ be the inclusion such that if $\iota_{j}(m)=\mathrm{m}$, then

$$
\mathrm{m}_{i}=\left\{\begin{array}{l}
m \text { for } i=j \\
0 \text { for } i \neq j
\end{array}\right.
$$

Let $\pi_{j}: \bigoplus_{i=1}^{n} M_{i} \rightarrow M_{j}$ be the projection: $\pi_{j}(\mathrm{~m})=\mathrm{m}_{j}$.

(4.1) Isomorphism of modules. There exists an $R$-isomorphism

$$
\phi: \operatorname{Ext}_{R}^{1}\left(\bigoplus_{i=1}^{n} M_{i}, N\right) \rightarrow \bigoplus_{i=1}^{n} \operatorname{Ext}_{R}^{1}\left(M_{i}, N\right)
$$

which maps $\alpha$ to $\left[\alpha \iota_{1}, \ldots, \alpha \iota_{n}\right]$, where the right action of $\iota_{j}$ on $\alpha$ is described in (2.1) (see [17, page 71]). Vice versa, if $\alpha_{i}$ is represented by the short exact sequence

$$
0 \rightarrow N \stackrel{\iota_{i}}{\longrightarrow} X_{\alpha_{i}} \stackrel{\rho_{i}}{\longrightarrow} M_{i} \rightarrow 0,
$$

then $\phi^{-1}\left(\left[\alpha_{1}, \ldots, \alpha_{n}\right]\right)$ is represented as the bottom short exact sequence, where $\nabla_{N}$ is the addition of $n$ elements from $N$, and $P$ is the pushout:

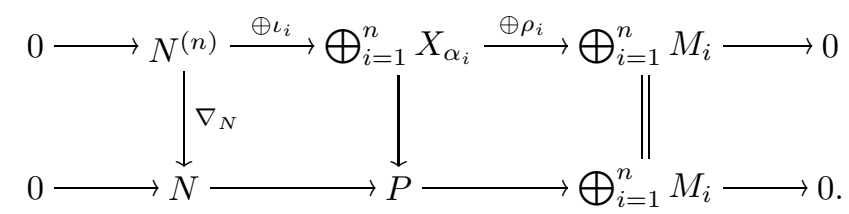


(4.2) Isomorphism of algebras. Let $\mathcal{B}=\operatorname{Hom}_{R}\left(\bigoplus M_{i}, \bigoplus M_{i}\right)$ and let $\mathcal{C}$ be the $R$-algebra of $n \times n$ matrices $B=\left(b_{i j}\right)$ where $b_{i j} \in \operatorname{Hom}_{R}\left(M_{i}, M_{j}\right)$. We have the following isomorphism:

$$
\psi: \mathcal{B} \rightarrow \mathcal{C}, \psi(b)=B^{T},
$$

where $B=\left(b_{i j}\right)$ and $b_{i j}=\pi_{j} \circ b \circ \iota_{i}$.

On the one hand, $\operatorname{Ext}_{R}^{1}\left(\bigoplus M_{i}, N\right)$ is a right $\mathcal{B}$-module, as described in (2.1). On the other hand, $\mathcal{C}$ acts on $\bigoplus_{i=1}^{n} \operatorname{Ext}_{R}^{1}\left(M_{i}, N\right)$ : let $\left[\alpha_{1}, \ldots, \alpha_{n}\right]$ be a row vector in $\bigoplus_{i=1}^{n} \operatorname{Ext}_{R}^{1}\left(M_{i}, N\right)$, and $C=\left(b_{i j}\right)$ be a matrix in $\mathcal{C}$. Then $\left[\alpha_{1}, \ldots, \alpha_{n}\right] C$ is given by matrix multiplication, where the right action of $b_{i j}$ on $\alpha_{i}$ is given as in (2.1). In the following, it is important to remember that a homomorphism acts on the right on short exact sequences, and on the left on elements. For example, if $f, g \in \operatorname{Hom}_{R}(M, M)$ for some module $M, m \in M$, and $\alpha \in \operatorname{Ext}_{R}^{1}(M, N)$, then we write $\alpha f g$ for $(\alpha f) g$ but $g \circ f(m)$ for $g(f(m))$.

(4.3) Proposition. Let $\phi$ and $\psi$ be the isomorphisms from (4.1) and (4.2). The equality $\phi(\alpha b)=\phi(\alpha) \psi(b)$ holds for every $\alpha \in \operatorname{Ext}_{R}^{1}\left(\bigoplus_{i=1}^{n} M_{i}, N\right)$ and $b \in \mathcal{B}$.

Proof. To see this, it is enough to show that equality holds for an element $b \in \mathcal{B}$ such that all but at most one entry $b_{i j}$ is zero, since the map $\phi$ is an $R$-homomorphism and therefore is additive.

Fix two integers $k$ and $l$. Fix an element $b \in \mathcal{B}$ such that $\psi(b)=B^{T}$, where $B=\left(b_{i j}\right)$, and $b_{i j} \neq 0$ if and only if $i=k$ and $j=l$. Let $\alpha \in \operatorname{Ext}_{R}^{1}\left(\bigoplus_{i=1}^{n} M_{i}, N\right)$ be represented by the short exact sequence:

$$
\alpha: 0 \longrightarrow N \longrightarrow X \stackrel{g}{\longrightarrow} \bigoplus_{i=1}^{n} M_{i} \longrightarrow 0 .
$$

We first compute the left-hand side of the equality in Proposition $4.3, \alpha b$ is given by

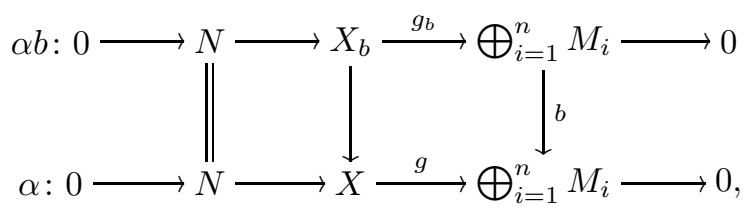

where $X_{b} \subset X \oplus\left(\bigoplus_{i=1}^{n} M_{i}\right)$ is given by

$$
X_{b}=\left\{(x, \mathrm{~m}) \mid g(x)=b(\mathrm{~m})=\left[0, \ldots, b_{k l}\left(\mathrm{~m}_{k}\right), 0 \ldots, 0\right]\right\},
$$

and $g_{b}((x, \mathrm{~m}))=\mathrm{m}$.

Now $\phi(\alpha b)=\left[\alpha b \iota_{1}, \ldots, \alpha b \iota_{n}\right]$, where

$$
\alpha b \iota_{j}: 0 \longrightarrow N \longrightarrow Y_{j} \stackrel{h_{j}}{\longrightarrow} M_{j} \longrightarrow 0,
$$

and where $Y_{j} \subset X_{b} \oplus M_{j} \subset X \oplus\left(\bigoplus_{i=1}^{n} M_{i}\right) \oplus M_{j}$ is given by

$$
Y_{j}=\left\{(x, \mathrm{~m}, m) \mid g(x)=\left[0, \ldots, b_{k l}\left(\mathrm{~m}_{k}\right), 0 \ldots, 0\right], \iota_{j}(m)=g_{b}((x, \mathrm{~m}))=\mathrm{m}\right\},
$$

and $h_{j}\left(\left(x, \mathrm{~m}, m_{j}\right)\right)=m$. It follows that

(1) $Y_{j} \cong N \oplus M_{j}$ for $j \neq k$, and therefore $\alpha f \iota_{j}=0$;

(2) $Y_{k} \cong\left\{\left(x, m_{k}\right) \in X \oplus M_{k} \mid g(x)=\left[0, \ldots, b_{k l}(m), \ldots, 0\right]\right\}$. 
In particular, $\phi(\alpha b)=\left[0, \ldots,(\alpha b) \iota_{k}, \ldots, 0\right]$.

To compute the right side of the equality, we first compute $\phi(\alpha)=\left[\alpha \iota_{1}, \ldots, \alpha \iota_{n}\right]$, where $\alpha \iota_{j}$ is given as

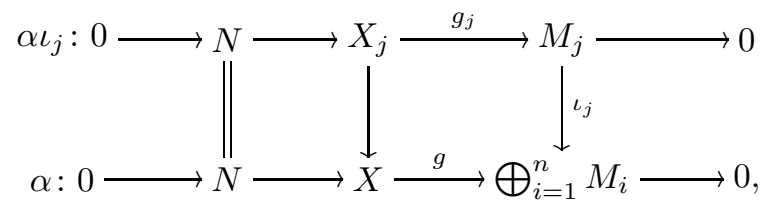

and where $X_{j}=\left\{\left(x, m_{j}\right) \in X \oplus M_{j} \mid g(x)=\iota_{j}\left(m_{j}\right)\right\}$ and $g_{j}\left(\left(x, m_{j}\right)\right)=m_{j}$. It follows that $\phi(\alpha) \psi(b)=\phi(\alpha) B^{T}=\left(0, \ldots,\left(\alpha \iota_{l}\right) b_{k l}, \ldots, 0\right)$, where the $k$-th entry is the only non-zero one.

All that is left to see is that $\left(\alpha \iota_{l}\right) b_{k l}=(\alpha b) \iota_{k}$. But there is an equality of homomorphisms $\iota_{l} b_{k l}=b \iota_{k}: M_{k} \rightarrow \bigoplus_{i=1}^{n} M_{i}$, so equality holds.

\section{ACKNOWLEDGMENTS}

We thank Roger Wiegand and Lars W. Christensen for their suggestions.

\section{REFERENCES}

1. Maurice Auslander, Finite type implies isolated singularity, Orders and their applications, Lecture Notes in Math., vol. 1142, Springer, Berlin, 1985. MR812487

2. Winfried Bruns and Jürgen Herzog, Cohen-Macaulay rings, Cambridge Studies in Advanced Mathematics, vol. 39, Cambridge University Press, Cambridge, 1993. MR1251956

3. R.-O. Buchweitz, G.-M. Greuel and F.-O. Schreyer, Cohen-Macaulay modules on hypersurface singularities. II, Invent. Math. 88 (1987), no. 1, 165-182. MR0877011 (88d:14005)

4. Andrew Crabbe, Daniel Katz, Janet Striuli, and Emanoil Theodorescu, Hilbert polynomials for the controvarient functor, preprint (2007).

5. David Eisenbud, Commutative algebra with a view toward algebraic geometry, Graduate Texts in Mathematics, vol. 150, Springer-Verlag, New York, 1995. MR97a:13001

6. Anders J. Frankild, Sean Sather-Wagstaff, and Roger Wiegand, Ascent of module structures, vanishing of ext, and extended modules, Mich. J. Math., to appear.

7. W. Hassler, R. Karr, L. Klingler, and R. Wiegand, Large indecomposable modules over local rings, J. Algebra 303 (2006), no. 1, 202-215. MR2253659

8. Wolfgang Hassler, Ryan Karr, Lee Klingler, and Roger Wiegand, Big indecomposable modules and direct-sum relations, Illinois J. Math. 51 (2007), no. 1, 99-122 (electronic). MR2346189

9. Indecomposable modules of large rank over Cohen-Macaulay local rings, Trans. Amer. Math. Soc. 360 (2008), no. 3, 1391-1406 (electronic). MR.2357700

10. Wolfgang Hassler and Roger Wiegand, Big indecomposable mixed modules over hypersurface singularities, Abelian groups, rings, modules, and homological algebra, Lect. Notes Pure Appl. Math., vol. 249, Chapman \& Hall/CRC, Boca Raton, FL, 2006, pp. 159-174. MR2229110 (2007h:13018)

11. Thomas W. Hungerford, Algebra, Graduate Texts in Mathematics, vol. 73, Springer-Verlag, New York, 1980, reprint of the 1974 original. MR600654

12. Daniel Katz and Emanoil Theodorescu, On the degree of Hilbert polynomials associated to the torsion functor, Proc. Amer. Math. Soc. 135 (2007), 3073-3082. MR2322736 (2008f:13021)

13. Lee Klingler and Lawrence S. Levy, Representation type of commutative Noetherian rings. I. Local wildness, Pacific J. Math. 200 (2001), no. 2, 345-386. MR.1868696

14. - Representation type of commutative Noetherian rings. II. Local tameness, Pacific J. Math. 200 (2001), no. 2, 387-483. MR1868697

15. - Representation type of commutative Noetherian rings. III. Global wildness and tameness, Mem. Amer. Math. Soc. 176 (2005), no. 832, viii+170. MR.2147090

16. Vijay Kodiyalam, Homological invariants of powers of an ideal, Proc. Amer. Math. Soc. 118 (1993), no. 3, 757-764. MR1156471 
17. Saunders Mac Lane, Homology, Classics in Mathematics, Springer-Verlag, Berlin, 1995, reprint of the 1975 edition. MR96d:18001

18. Joseph J. Rotman, An introduction to homological algebra, Pure and Applied Mathematics, vol. 85, Academic Press Inc. [Harcourt Brace Jovanovich Publishers], New York, 1979. MR.538169

Department of Mathematics, University of Nebraska, Lincoln, Nebraska 68588

Current address: Department of Mathematics, Syracuse University, Syracuse, New York 13210

E-mail address: amcrabbe@syr.edu

Department of Mathematics, University of Nebraska, Lincoln, Nebraska 68588

Current address: Department of Mathematics, Fairfield University, Fairfield, Connecticut 06824

E-mail address: jstriuli@mail.fairfield.edu 